\title{
Label-free volumetric imaging of conjunctival collecting lymphatics ex vivo by optical coherence tomography lymphangiography
}

\author{
Peijun Gong $^{1 *}$ (1) | Dao-Yi Yu ${ }^{2,3}$ | Qiang Wang ${ }^{1}$ | Paula K. Yu ${ }^{2,3}$ | Karol Karnowski ${ }^{1}$ (D) | \\ Morgan Heisler $^{4}$ | Ashley Francke ${ }^{4}$ | Dong An $^{2,3}$ | Marinko V. Sarunic ${ }^{4}$ | David D. Sampson ${ }^{1,5}$ (D)
}

\author{
${ }^{1}$ Optical+Biomedical Engineering Laboratory, \\ Department of Electrical, Electronic and Computer \\ Engineering, The University of Western Australia, \\ Perth, WA, Australia \\ ${ }^{2}$ Centre for Ophthalmology and Visual Science, \\ The University of Western Australia, Perth, WA, \\ Australia \\ ${ }^{3}$ Lions Eye Institute, Nedlands, WA, Australia \\ ${ }^{4}$ Biomedical Optics Research Group, School of \\ Engineering Science, Simon Fraser University, \\ Burnaby, BC, Canada \\ ${ }^{5}$ University of Surrey, Guildford, Surrey, UK \\ *Correspondence \\ Peijun Gong, Optical+Biomedical Engineering \\ Laboratory, Department of Electrical, Electronic \\ and Computer Engineering, The University of \\ Western Australia, 35 Stirling Highway, Perth WA \\ 6009, Australia. \\ Email: peijun.gong@uwa.edu.au
}

We employ optical coherence tomography (OCT) and optical coherence microscopy (OCM) to study conjunctival lymphatics in porcine eyes ex vivo. This study is a precursor to the development of in vivo imaging of the collecting lymphatics for potentially guiding and monitoring glaucoma filtration surgery. OCT scans at $1300 \mathrm{~nm}$ and higher-resolution OCM scans at $785 \mathrm{~nm}$ reveal the lymphatic vessels

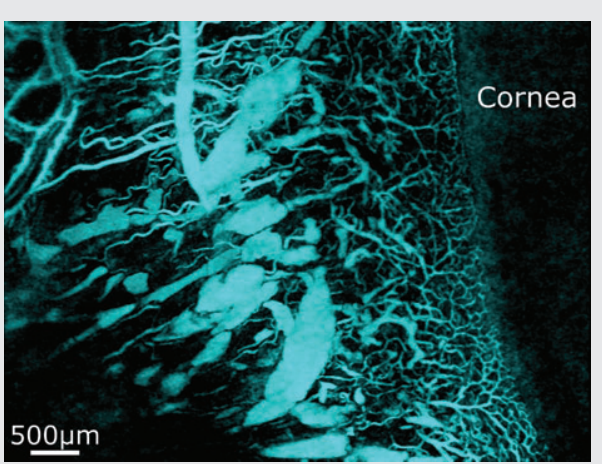
via their optical transparency. Equivalent signal characteristics are also observed from blood vessels largely free of blood (and devoid of flow) in the ex vivo conjunctiva. In our lymphangiography, vessel networks were segmented by compensating the depth attenuation in the volumetric OCT/OCM signal, projecting the minimum intensity in two dimensions and thresholding to generate a threedimensional vessel volume. Vessel segmentation from multiple locations of a range of porcine eyes $(n=21)$ enables visualization of the vessel networks and indicates the varying spatial distribution of patent lymphatics. Such visualization provides a new tool to investigate conjunctival vessels in tissue ex vivo without need for histological tissue processing and a valuable reference on vessel morphology for the in vivo label-free imaging studies of lymphatics to follow.

\section{KEYWORDS}

aqueous veins, blood vessels, conjunctiva, glaucoma, lymphangiography, lymphatics, optical coherence tomography, speckle decorrelation

\section{1 | INTRODUCTION}

The lymphatic system provides an important drainage function in biological tissue. Lymphatic flow removes excess interstitial fluid to support fluid/macromolecular balance, lipid absorption and immune function. Lymph originates from the collection of interstitial fluid in the initial lymphatics, termed lymphatic capillaries, which are 10 to $60 \mu \mathrm{m}$ in diameter [1]. These capillaries merge into larger collecting lymphatics $>200 \mu \mathrm{m}$ in diameter containing valve structures [2], passing through one or multiple lymph nodes (1-10 $\mathrm{mm}$ in size) [1]. The valve structures in the collecting lymphatics, termed lymphangions, prevent backflow and direct the lymph to the blood circulatory system via the lymphatic trunks or the sac-like structure (cisterna chyli) in the thoracic duct [1]. 
Imaging the lymphatics is important in investigating tissue physiologies, pathologies and therapies [3, 4], but imaging the initial lymphatics is currently challenging both in vivo and ex vivo. Various lymphangiography techniques have been developed for imaging lymphatics in vivo, based on near-infrared fluorescence imaging (indocyanine green lymphangiography), ultrasound, computed tomography, magnetic resonance imaging and positron emission tomography $[3,5,6]$. All are limited to large lymphatic vessels (on the millimeter scale) due to limited spatial resolution and typically require injection of exogenous contrast agents to label vessels. Photoacoustic imaging is an emerging in vivo lymphangiography technique that may provide higher resolution [3, 7], also aided by exogenous contrast agents. Histology is an alternative important source of information [8], but can be particularly challenging for lymphatics. Due to the delicate vessel structures, it is susceptible to artifacts, and requires laborious, painstaking work for effective threedimensional (3D) imaging. Thus, a label-free, highresolution 3D imaging capability is an attractive approach to investigate the lymphatic system at the level of capillaries.

An example of where it is important to have a greater understanding of lymphatics than currently available is in the conjunctiva. Conjunctival lymphatics form an incompletely understood part of the eye's pressure relief mechanism that is involved in glaucoma filtration surgery [8]. Glaucoma is one of the leading causes of blindness, with 79.6 million people estimated to be affected worldwide by 2020 [9]. Most glaucoma patients have elevated intraocular pressure (IOP), where the increased outflow resistance of the aqueous humor is thought to be a contributing factor. Prolonged exposure to high IOP can lead to damage of the retina and vision loss. Glaucoma filtration surgery seeks to neutralize the elevated IOP by creating an additional drainage pathway for the aqueous humor through a channel leading to the subconjunctival tissue, forming a filtering bleb (ie, localized pooling of the aqueous humor). The mechanism of drainage from the bleb is not fully elucidated, but previous studies have suggested that the integrity and density of conjunctival lymphatics are key for the longevity of the created drainage pathway and, thus, for good treatment outcomes [8]. Investigation of this and other clinical and biological challenges associated with lymphatics requires a better imaging technique.

Optical coherence tomography (OCT) is a label-free imaging technique that measures depth-resolved tissue reflectance, achieving 3D imaging at micrometer-scale resolutions (typically $5-20 \mu \mathrm{m}$ ). A family of OCT extensions have been developed for imaging blood vessels in vivo by measuring the dynamic changes of the amplitude and/or phase of the OCT signal induced by motion of the strong scatterers in blood [10, 11]. These methods, collectively referred to as OCT angiography, have been applied to imaging blood vessels in various tissues, such as the eye, brain and skin [11-16]. In addition, OCT has been investigated for imaging the lymphatic vessels in vivo, with a novel and distinct contrast mechanism, namely, taking advantage of the characteristically low OCT signal resulting from the lowscattering, transparent lymph $[12,17,18]$. The pilot combination of these two families of techniques has shown the feasibility of OCT for simultaneous imaging of both vessel networks in human skin tissue in vivo [17]. Currently, there is limited adoption of such techniques for imaging vessels in the conjunctiva in vivo, with a single work demonstrating this in the wider context of graft-vs-host disease [19].

As a precursor to imaging in the more complex scenario of dynamic living tissue, it is helpful to understand the morphology of the conjunctival vessel structures measured under less challenging conditions. We, thus, present a study of conjunctival lymphatics imaged ex vivo using OCT lymphangiography. Equivalent to the in vivo case, we observe low OCT signal without shadowing in the lymphatic vessels of the ex vivo tissue. The same signal feature is observed for blood vessels. We identify the vessel network using an automatic algorithm that identifies local regions of low signal after compensating for the depth-dependent attenuation of the OCT signal. We imaged excised porcine eyes $(n=21)$, visualizing the vessels at multiple locations of the conjunctiva in each eye. The resulting images from a commercial high-resolution (axial: $5.5 \mu \mathrm{m}$ in air; transverse: $13 \mu \mathrm{m}$ ) $1300 \mathrm{~nm}$ OCT scanner and a home-built ultrahigh-resolution (axial: $1.5 \mu \mathrm{m}$ in air; transverse: $1.6 \mu \mathrm{m}$ ) $785 \mathrm{~nm}$ optical coherence microscopy (OCM) scanner visualize the morphological characteristics of the vessel networks at different resolutions. Thereby, we provide the most detailed and comprehensive OCT-based visualization of the vessel structures in the conjunctiva reported to date. Although ex vivo, there is no automatic differentiation of blood and lymphatic vessels, morphological features, such as valves and vessel shapes, may be used to identify the lymphatics. Such visualizations provide valuable reference vessel morphology, which is a necessary and useful prior step before moving to in vivo label-free imaging of lymphatic vessels.

\section{2 | OCT LYMPHANGIOGRAPHY METHODS}

\subsection{Ex vivo lymphatic and blood vessel characteristics in OCT signal}

In the in vivo tissue environment, lymph and blood produce distinctly different characteristics in the OCT signal and in the corresponding speckle decorrelation, which is calculated using a pair of sequential OCT B-scans acquired from the same tissue location [15, 17]. Lymphatic vessels present very low OCT signal, approaching the noise floor, due to their transparency to near-infrared OCT light, whilst blood vessels present lower signal than the surrounding highscattering tissue due to strong forward scattering (ie, low 

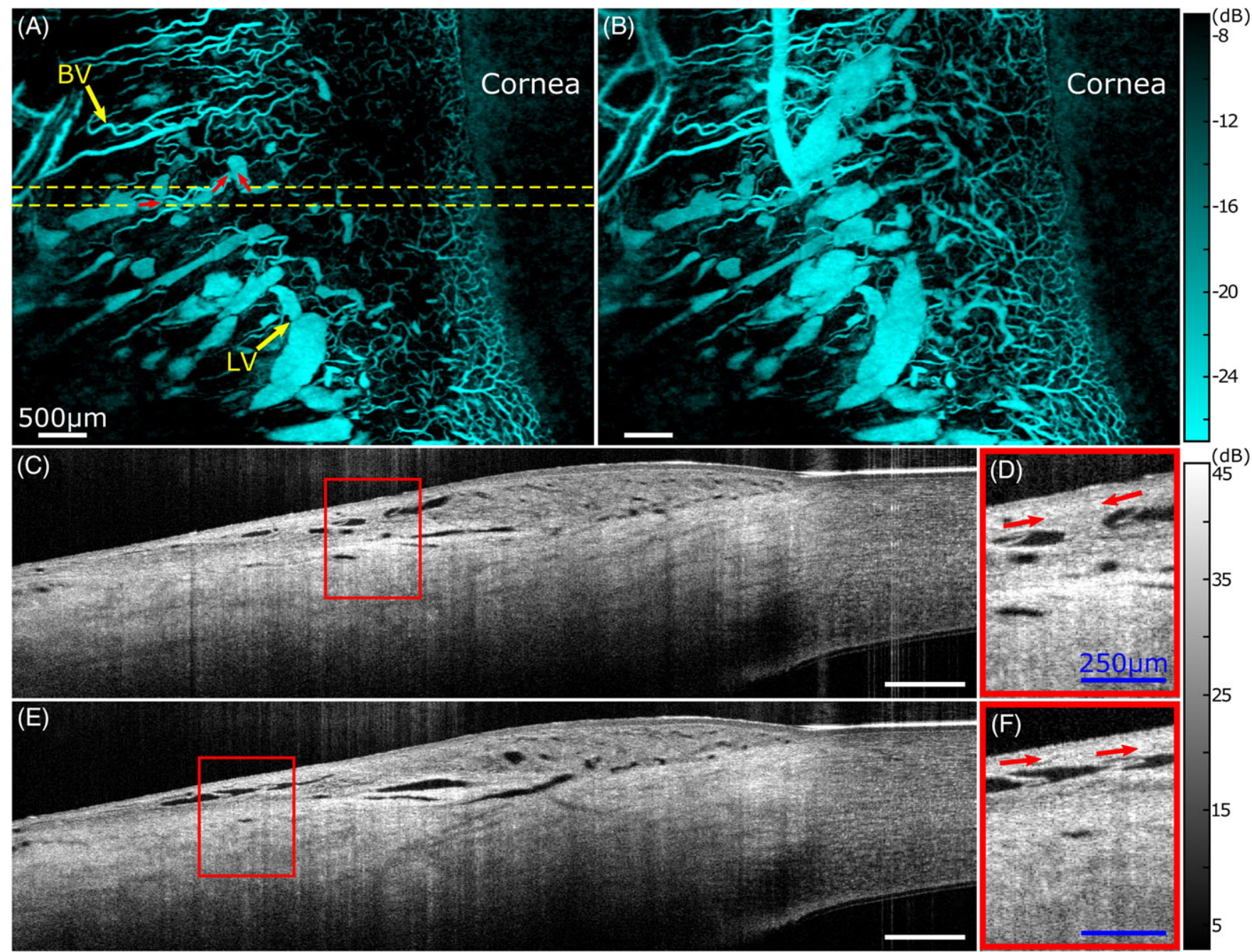

FIGURE 1 OCT imaging of lymphatic and blood vessels of ex vivo conjunctiva in Sample 1. (A) and (B) Projection images of vessels, respectively, from the surface to 200 and $350 \mu \mathrm{m}$ deep. The cornea is indicated by the label, and blood and lymphatic vessels by the labels BV, blood vessel; LV, lymphatic vessel. Yellow dashed lines in (A) indicate locations of scans shown in (C) and (E). (C) and (E) OCT B-scans with the regions outlined by the red rectangles magnified in (D) and (F). Red arrows in (A), (D), and (F) indicate the original lymph flow directions. White scale bars: $500 \mu \mathrm{m}$; Blue scale bars: $250 \mu \mathrm{m}$

backscattering), but typically much higher than the noise floor for human burn scar tissue. As for speckle decorrelation, lymphatic vessels show high decorrelation within the vessels due to the very low signal (ie, largely uncorrelated noise is detected), whilst blood vessels are characterized by flow-induced high decorrelation. This decorrelation also extends to the tissue areas underlying the vessels, known as shadow or projection artifacts [20-22]. These characteristics allow the use of a previously proposed method to differentiate and segment both vessel networks in skin tissue in vivo: thresholding of the OCT signal to segment the lymphatic vessels and weighting of the decorrelation with the OCT signal intensity to image the blood vessels [17].

In the ex vivo conjunctiva in this study, we observed vessel structures with low OCT signal, equivalent to the in vivo case for lymphatic vessels, as shown in the en face minimum intensity projection image in Figure 1A,B from an eye sample (Sample 1). The results were obtained by applying the method described in Section 2.2 to the scans acquired by the OCT scanner described in the Section 2.3. The vessels are projected from the tissue surface to depths of, respectively, 200 and $350 \mu \mathrm{m}$. All depths in tissue given in this paper are physical distances, as measured by OCT assuming an average refractive index of 1.4. These vessel structures typically present two distinct morphological features: thick and uneven or thin and even. Examples of each, labeled LV and $\mathrm{BV}$, respectively, are indicated by the yellow arrows in Figure 1A.

Closer examination of the thick vessel structures reveals uneven diameter along the vessel segments together with frequent local narrow discontinuity, which is due to the presence of valve structures, indicating that they are lymphatic pre-collectors. Representative B-scans containing such structures are shown in Figure 1C,E, taken from the locations indicated by the yellow dashed lines in Figure 1A. These structures typically have a thin, "semilunar" shape, such as those magnified in Figure 1D,F, corresponding well to the expected shape of valve leaflets. We also observe neighboring valves structures in the same vessel forming a functional unit, the lymphangion, such as in Figure $1 \mathrm{~F}$. The valve 
leaflets evident in Figure 1D,F agree well with the observed vessel discontinuity in previous OCT lymphangiography studies in vivo [19]. In addition, the orientation of the valve leaflets (functioning to prevent backflow) can be used to determine the local lymph flow direction in the original living tissue, indicated by the red arrows in Figure 1D,F. These flow directions can be then mapped in the en face image in Figure 1A to provide the overall flow direction, such as shown by the red arrows in Figure 1A.

In contrast, the thin vessels present very uniform diameters, as shown in Figure 1A,B, without the presence of the valve-like structures seen in the thick vessels. Their features suggest that they are the blood vessels in the conjunctival tissue. To further support the classification of these vessels, we used a high-resolution OCM scanner, described in Section 2.3, to examine the vessel structures. The OCM scans confirm the consistent thick and thin vessels seen in the OCT scans, and are presented in Section 3. The equivalently low OCT signal from both the lymphatic and blood vessels prevents their differentiation based on the OCT signal and speckle decorrelation, as used in the in vivo case [17], but allows the segmentation of both vessel networks using the same approach that we used previously, described in the following section.

\section{2 | Vessel segmentation}

Our method utilizes the characteristically low OCT signal in the vessels to segment them from the background tissue [17]. The depth-dependent OCT signal can be modeled by the single-scattering model for a relatively homogeneous sample, which follows the exponential decay of the signal along the depth into the tissue [23-25], as shown in Eq. (1):

$$
I(z) \propto F(z) S(z) \rho e^{-2 \mu_{t} z},
$$

where the OCT signal, $I(z)$, is the square of the modulus of the complex amplitude of the OCT signal resulting from inverse Fourier transformation of the signal from the spectral-domain scanner; and $\rho e^{-2 \mu_{t} z}$ represents the exponential decay with $\rho$ and $\mu_{t}$ being the reflectance at the surface and the attenuation coefficient of the tissue, respectively. This exponential decay is further modulated by the system-dependent factors, including the confocal function, $F(z)$, mainly due to the focusing optics in the sample arm and the sensitivity fall-off function, $S(z)$, due to the finite spectral resolution of the spectrometer in spectraldomain OCT. The vessels then introduce a local decrease of the signal almost to the noise floor, superimposed on the global exponential depth decay of the OCT signal.

The use of the low OCT signal to segment the vessels by thresholding is subject to significant segmentation artifacts due to the attenuation inherent in Eq. (1). To mitigate such artifacts, our method specifically compensates for the attenuation based on the single-scattering model. The first step is to correct for the response functions $F(z)$ and $S(z)$ by dividing the measured OCT signal by the calibration signal acquired from a low-scattering phantom of polystyrene microspheres in deionized water as:

$$
I_{C}(z)=\frac{I(z)}{I_{0}(z)} \propto \frac{F(z) S(z) \rho e^{-2 \mu_{t} z}}{F(z) S(z) \rho_{0} e^{-2 \mu_{0} z}} \propto \frac{\rho}{\rho_{0}} e^{-2\left(\mu_{t}-\mu_{t 0}\right) z},
$$

where $I_{C}(z)$ is the corrected OCT signal; and $I_{0}(z)$ is the calibration signal based on Eq. (1) with $\rho_{0}$ and $\mu_{t 0}$ being the surface reflectance and the attenuation coefficient of the calibration phantom. $\mu_{t 0}$ is estimated from Mie theory and is typically smaller than $0.1 \mathrm{~mm}^{-1}$ and so neglected due to the low concentration of microspheres (diameter: $0.5 \mu \mathrm{m}$; Polybead, Polysciences, Inc., Warrington, Pennsylvania) in the calibration liquid. The corrected OCT signal is fitted to the exponential model in Eq. (2) via weighted least-squares regression, giving an estimated attenuation coefficient, $\mu_{t}$, for each A-scan. The use of the OCT signal intensity as the weights in the weighted least-squares regression minimizes the impact of the local low signal in the vessels on the fitting and provides an estimation of the global depth attenuation. The corrected OCT signal is then multiplied by the compensation function $e^{2\left(\mu_{t}-\mu_{0}\right) z}$. The compensated OCT signal exhibits nearly constant baseline signal intensity vs depth, which is proportional to $\rho / \rho_{0}$, with the vessels presenting much lower signal.

The tissue surface is detected with a Canny edge detector and the vessels are segmented in two dimensions (2D) by finding the minimum signal intensity from the surface to a certain depth at each lateral location of the compensated scan, forming a 2D projection image. One disadvantage of this $2 \mathrm{D}$ segmentation is the apparent overlapping of vessels at different depths, presenting as misleading connections in the projection image. Therefore, our method also segments the vessels in 3D without projection by thresholding the compensated OCT signal with a single threshold for the whole scan. These 2D and 3D images comprise both the blood and lymphatic vessels, which can be distinguished manually using the vessel shapes. Note that the 3D segmentation of the ex vivo blood vessel network provides a new approach for true 3D visualization; whereas, the common methods for imaging blood vessels in vivo, such as speckle decorrelation, are typically not capable of true 3D imaging due to the presence of shadow artifacts.

\section{3 | OCT scanning of conjunctiva ex vivo}

Porcine eyes $(n=21)$ were enucleated and prepared for OCT and OCM imaging ex vivo. The eyes were kept in saline prior to imaging, with the imaging completed within $\sim 3$ to 4 hours of enucleation in order to minimize the possible decay of vessel patency over time in the ex vivo tissue. A custom-designed sample holder was used to fix the eyeball, as shown in Figure 2A, allowing easy adjustment of the orientation for OCT scanning of multiple regions of the conjunctiva. Care was taken during tissue preparation and 


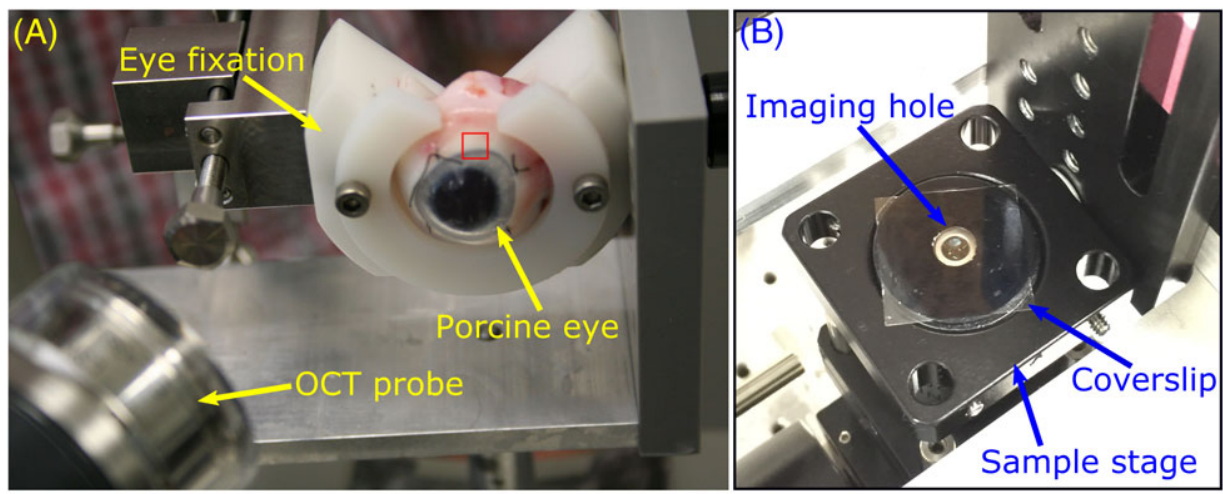

(C)

\begin{tabular}{l|c|c}
\hline Scanner & TELESTO II OCT & Home-built OCM \\
\hline Central wavelength $(\mathrm{nm})$ & 1300 & 785 \\
\hline Axial resolution in air $(\mu \mathrm{m})$ & 5.5 & 1.5 \\
\hline Transverse resolution $(\mu \mathrm{m})$ & 13 & 1.6 \\
\hline Axial scan rate $(\mathrm{kHz})$ & $48 / 76$ & 20 \\
\hline
\end{tabular}

FIGURE 2 Scanning setup and specifications of scanners for ex vivo conjunctival imaging. (A) Photograph of the OCT scanning setup. (B) Photograph of the OCM sample stage. (C) Specifications of the OCT and OCM scanners

imaging to make sure that areas for imaging were not contacted so as to avoid artificial pressure which could potentially impact the vessel patency. Saline drops were constantly applied to the eyeballs to keep the tissue hydrated during the scanning session. The scanning protocol was approved by Animal Ethics Committee of The University of Western Australia.

Two scanners were used in this study: a commercial OCT scanner and an ultrahigh-resolution home-built OCM scanner. We used the spectral-domain OCT scanner (TELESTO II, Thorlabs Inc., Newton, New Jersey) to scan one or multiple regions of conjunctival tissue in each eye. This scanner is based on a Michelson interferometer setup and the details have been described previously [15]. It has an axial and lateral imaging resolution of, respectively, $5.5 \mu \mathrm{m}$ (in air) and $13 \mu \mathrm{m}$, with a central wavelength of $1300 \mathrm{~nm}$. Scans were acquired at an axial scan rate of $48 \mathrm{kHz}$ or $76 \mathrm{kHz}$ with imaging field of view (FOV) of $3 \times 3 \mathrm{~mm}$ or $6 \times 4.5 \mathrm{~mm}$. For several eyes, multiple OCT FOVs were recorded from neighboring tissue regions and synthesized to investigate the wide-field imaging capability.

To investigate the more detailed vessel features, we also used a home-built spectral-domain OCM scanner to image multiple conjunctiva tissue regions in two porcine eyes (Sample 2 and 3). This bench-top system utilizes a supercontinuum light source (SuperK Extreme EXR-1, NKT Photonics, Birkerød, Denmark) to generate spectrally filtered broadband light with a $3 \mathrm{~dB}$ bandwidth of $200 \mathrm{~nm}$ at a central wavelength of $785 \mathrm{~nm}$, leading to a measured axial imaging resolution of $1.5 \mu \mathrm{m}$ [26]. To extend the limited depth of field of Gaussian beam illumination associated with the high-numerical aperture in typical OCM systems, our scanner uses Bessel beam illumination generated by a spatial light modulator (Pluto NIR II-HR, Holoeye Photonics AG, Berlin, Germany). This illumination passes through a small circular hole and a glass coverslip acting as the sample stage (Figure 2B) and onto the eyeball placed on the coverslip. The OCM system uses a Mach-Zehnder interferometer setup with Gaussian beam detection mode achieving a measured lateral resolution of $1.6 \mu \mathrm{m}$, as described in [26]. The scanner operated at a scanning speed of $20 \mathrm{kHz}$ with a FOV of $1.5 \times 1.5 \mathrm{~mm}$ for all scans. The key specifications of the two scanners are summarized in Figure 2C.

The acquired OCT scans were then processed using our aforementioned method, coded in MATLAB (R2016a, The MathWorks, Inc., Natick, Massachusetts), to image the vessel network in both 2D and 3D. In order to achieve widefield imaging by combining scans from neighboring tissue regions of the same eyes, the generated 2D vessel images from each location were manually compared to identify common vessel patterns. These vessel patterns were then used to register the vessel images by $2 \mathrm{D}$ rotation and translation, and to merge the images from multiple $(n=4-6)$ OCT FOVs into a single wide-field image.

\section{I OCM AND OCT LYMPHANGIOGRAPHY IMAGES OF CONJUNCTIVA}

In this section, we present OCM, OCT and derived parametric images of the conjunctival tissue, visualizing morphological features in 2D and 3D. A proof-of-principle segmentation of lymphatic vessels based on morphological vessel features is also presented. In addition, we show an example of imaging the aqueous veins in deep tissue after excisional removal of the thin conjunctiva. 


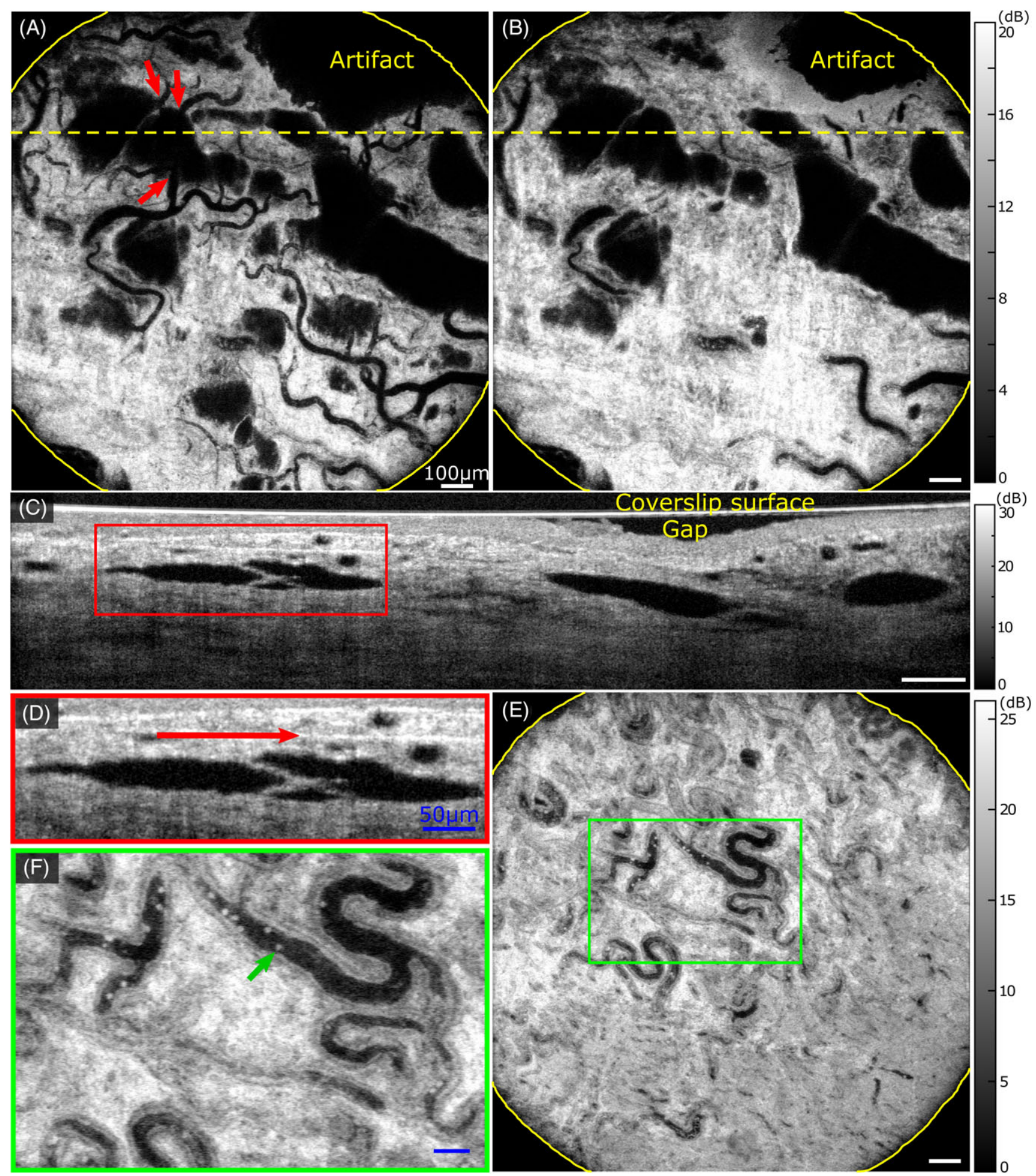

FIGURE 3 OCM images of conjunctival vessels. (A) and (B) Projection images of vessels in the depth ranges of 40 to $110 \mu \mathrm{m}$ and 90 to $110 \mu \mathrm{m}$ below the coverslip from Sample 2. Yellow dashed lines show the location of the B-scan presented in (C). An artifact caused by an air gap between the coverslip and sample is labeled. (C) OCT B-scan with a valve structure. The artifact in (A) and (B) is labeled and the red box is magnified in (D) with the flow direction indicated by the red arrow. (E) Projection image of vessels in the depth range of 50 to $80 \mu \mathrm{m}$ below the coverslip from Sample 3 with the region in the green rectangle magnified in $(\mathrm{F})$. The green arrow shows a red blood cell. White scale bars: $100 \mu \mathrm{m}$; Blue scale bars: $50 \mu \mathrm{m}$

\subsection{OCM imaging of conjunctival vessels}

Figure 3 shows a typical example of OCM images (en face and cross-sectional) of the conjunctiva showing vessel structures. The yellow solid lines in Figure 3 outline the edges of the imaging hole of the sample stage (as shown in Figure 2B) included in the imaging FOV. The minimum intensity projection images of the vessels in Figure $3 \mathrm{~A}, \mathrm{~B}$ from Sample 2 are, respectively, from the depth ranges of 
40 to $110 \mu \mathrm{m}$ and 90 to $110 \mu \mathrm{m}$ below the coverslip surface in the same OCM scan. This scan shows a region with a gap between the tissue surface and the coverslip surface (labeled in Figure 3C), leading to the projection of the labeled artificially low-signal region in the top right corner. Figure 3A captures more vessels over a larger depth range, but the projection leads to misleading visual information, such as the false vessel connections indicated by the red arrows, which are eliminated by using the smaller projection depth range in Figure 3B. These high-resolution images distinctly show the thick uneven and thin even vessels, consistent with the OCT scans. The thick vessels typically contain the thin semilunar valve structures, as shown by the B-scan (Figure 3C) from the location indicated by the dashed lines in Figure 3A,B. In the magnified image in Figure 3D, the flow direction in the original living tissue is predicted by the red arrow based on the orientation of the valve leaflets. Such lymphatic valve leaflets comprise an extracellular matrix core with a specialized anchoring endothelium on both sides, with their total thickness estimated to be $\sim 9 \mu \mathrm{m}$ in Figure 3D. Previous studies have reported that the thickness of lymphatic valve leaflets in rabbit lungs ranges from $0.5 \mu \mathrm{m}$ to more than $6 \mu \mathrm{m}$ [27], which are thinner than those in Figure 3D.

The valve structures are absent from the thin even vessels in the OCM scans, which suggests that they are blood vessels. In addition, we noticed the presence of sparse circular-shaped strong scatterers inside the lumen of thin vessels, as shown in Figure 3E from another OCM scan (Sample 3), which are absent in the thick vessels. These scatterers have an estimated diameter of $\sim 6 \mu \mathrm{m}$ in the magnified image in Figure $3 \mathrm{~F}$, and are very likely the remaining blood cells inside the blood vessels. This further indicates that the thin vessels are blood vessels containing sparse, static red blood cells in ex vivo conjunctival tissue, leading to their low OCM and OCT signal levels equivalent to those of lymphatic vessels. The high-imaging resolution of OCM allows the examination of these vessels in greater detail, but limited to a smaller FOV.

\section{2 | OCT imaging of conjunctival vessels in 2D}

A 2D image, comprising 4 stitched images of the segmented vessels in the conjunctival tissue of a porcine eye (Sample 4 ), is shown in Figure 4. The 2D projection images (Figure 4A) span $400 \mu \mathrm{m}$ into the tissue. This region of conjunctiva shows a rich distribution of lymphatic vessels combined with a number of thin blood vessels. The lymphatic vessels have prevalent valve structures evident in different vessel sizes with three examples shown by the B-scans in Figure 4B-D, from the locations between the red arrowheads in Figure 4A. Figure 4B is from a vessel with very small size in the axial direction, which has an estimated axial inner diameter of $\sim 10$ to $20 \mu \mathrm{m}$. Inside this vessel lumen, the valve leaflets present a space (indicated by the green arrow in Figure 4B) as narrow as $\sim 5 \mu \mathrm{m}$ to the vessel wall, which imposes a high requirement on the system imaging resolution to clearly resolve such structures. The lateral size of this vessel is estimated to be $\sim 58 \mu \mathrm{m}$ (Figure $4 \mathrm{~A}$ ), indicating that this vessel has a flat shape in the vessel cross section in the ex vivo tissue. By contrast, the valves in the larger vessels, such as in Figure 4C,D, are easier to capture, as the leaflets present a large space to the vessel walls. Overall, the crosssectional thickness of the valve leaflets in these three vessels is estimated to be in the range 9 to $18 \mu \mathrm{m}$, which is thicker than that in Figure 3D. When these valve leaflets are oriented largely along the depth direction, their corresponding high-signal leads to the local high-projection values (ie, black in Figure 4A) and, thus, leads to discontinuity in the vessels thereby forming the "bamboo stem" pattern in the projection image, as indicated by the magenta arrows in Figure 4A.

The conjunctival lymphatic vessels present a large degree of variation in distribution across the multiple porcine eyes examined in this study. For example, the segmented conjunctival vessels from up to $350 \mu \mathrm{m}$ below the tissue surface from another eye (Sample 5) show a lower density of lymphatic vessels in Figure 5, but a higher density of blood vessels, compared to Figure 4A. The lymphatic vessels at this location have a sparse distribution with irregular shapes and very uneven diameters along the vessel orientation.

Whereas there are relatively rich distributions of lymphatic vessels in Figures 4 and 5, the conjunctival tissue of Sample 6, presented in Figure 6 (to $225 \mu \mathrm{m}$ below the tissue surface), shows a lack of obvious lymphatic vessels. Such variations may be due to heterogeneity in the spatial distribution of lymphatic vessels among different samples and/or different circumferential locations within a sample, or the result of locally collapsed lymphatic vessels in the ex vivo tissue. This latter case would need further histology to validate. In addition, the overlapping vessels in the $2 \mathrm{D}$ projection image in Figure 6A present misleading visual artifacts, for example, at the locations indicated by the magenta arrows. By comparison with the color-coded depth of the top surface of the vessels, shown in Figure 6B, the visual artifacts in Figure 6A lead to the false connection of small vessels to large vessels and missing connections of the small vessels above large vessels apparent in Figure 6A. Therefore, knowledge of the depth of the vessels is also important for correct interpretation of the vessel organization in $2 \mathrm{D}$ projection images.

\subsection{OCT imaging of conjunctival vessels in 3D}

We segmented the vessels in all 3D-OCT scans by thresholding the attenuation-compensated signal, with two examples shown in Figure 7. Figure 7A shows the en face image of the color-coded depth of the top surface of the 3D segmented vessels corresponding to the scan presented in Figure 1B (Sample 1). Whereas this depth image can indicate the start depth and the lateral size of the vessels, it does 


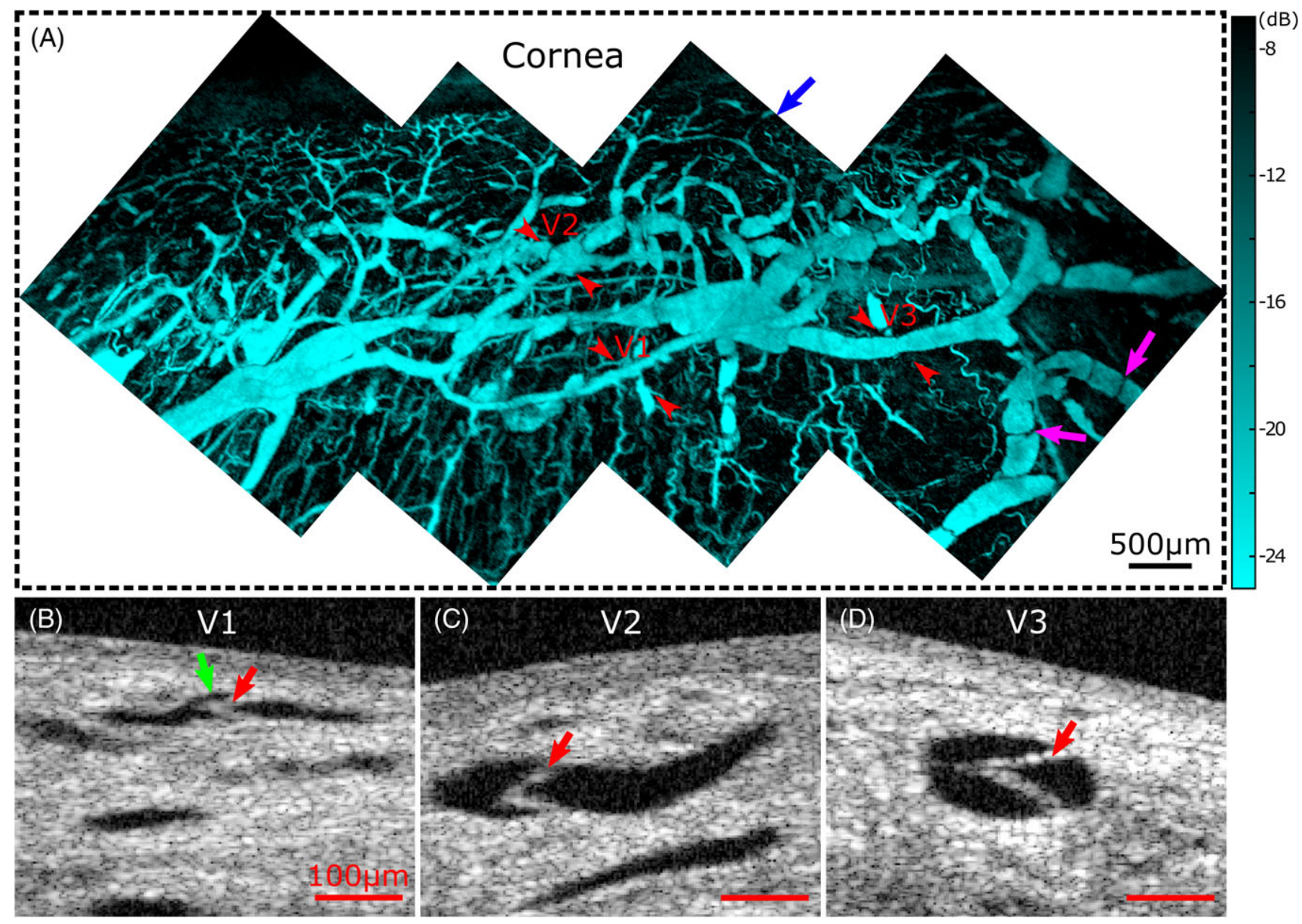

FIGURE 4 OCT imaging of conjunctival vessels in Sample 4. (A) Projection image of vessels from the surface to $400 \mu \mathrm{m}$ deep formed by montaging 4 scans. The cornea is labeled at the top of the image in this orientation. Blue arrow: OCT FOV for 3D visualization shown in Figure 7. Magenta arrows: vessel discontinuity. Arrowheads and labels V1, V2 and V3 show locations of OCT B-scans shown in (B-D). Red arrows: lymphatic valve leaflets. Green arrow: space between vessel wall and valve leaflet. Red scale bars: $100 \mu \mathrm{m}$. Colormaps in (B-D) show OCT signal from 3 to $\sim 45 \mathrm{~dB}$

not provide the extent of the vessels along the depth direction. Thus, we detected the surface of the 3D segmented vessels, and visualized them in $3 \mathrm{D}$, as shown in Visualization 1 (Video S1, Supporting Information) with rendering as indicated in Figure 7B. The 3D visualization allows an intuitive interpretation of the vessel morphology, revealing, for example, the flat shape of the large lymphatic vessel indicated by the red arrow in Figure 7A,B.

The vessels in the OCT FOV indicated by the blue arrow in Figure 4A of Sample 4 have their vessel depth colorcoded in Figure 7C. The two vessels marked by the red arrows do not seem to connect to the large lymphatic vessel

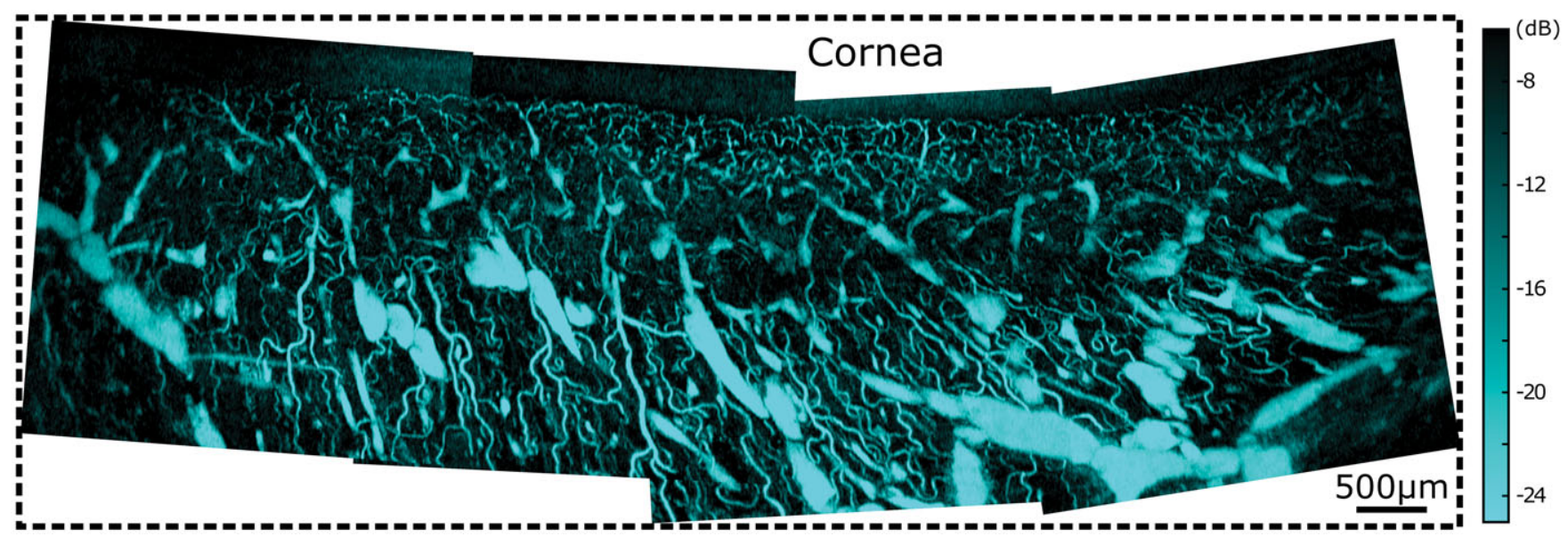

FIGURE 5 Projection image formed by segmenting vessels from OCT imaging of conjunctiva in Sample 5. Projection from the surface to $350 \mu \mathrm{m}$ deep, formed by montaging 4 images 

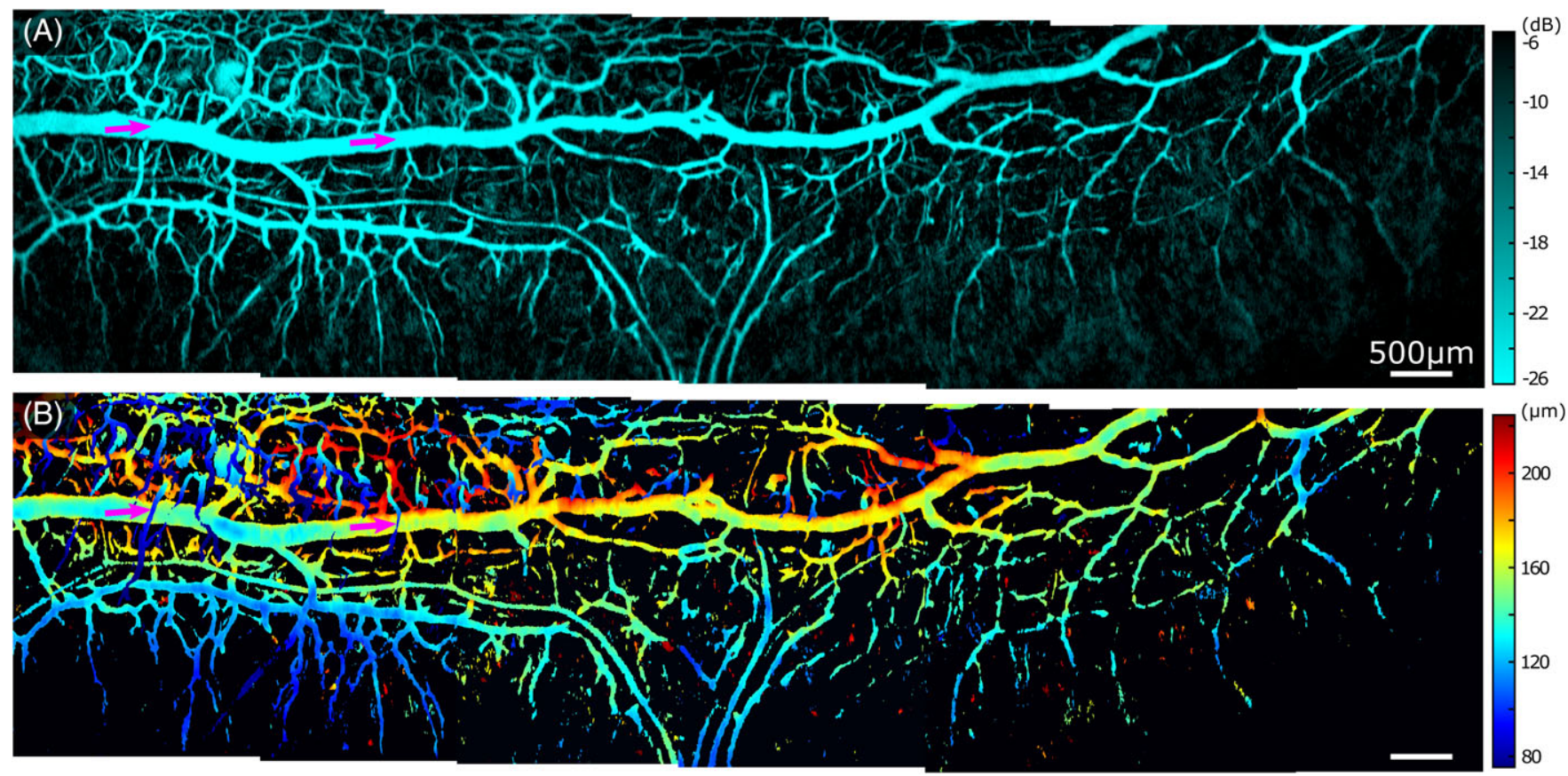

FIGURE 6 Projection images from OCT imaging of conjunctival vessels in Sample 6. (A) Projection image of vessels from the surface to $225 \mu \mathrm{m}$ deep. (B) Color-coded depth of the top surface of the vessels. Scale bars: $500 \mu \mathrm{m}$. Images formed by montaging 6 images

in between them, indicated by the green arrow, due to their mismatch in depth. The 3D visualization (Visualization 2, Video S2) confirms this disconnection. In addition, the connection of these two vessels (indicated by the red arrows) located beneath the flat large lymphatic vessel (green arrow) is validated in such volumetric imaging. The $3 \mathrm{D}$ vessel imaging capability provided by our method avoids the misleading visual information provided by $2 \mathrm{D}$ projections of vessels. In addition, volumetric segmentation allows the quantification of the volume fraction of the vessel structures as the ratio of the sum of the vessel volume to the total tissue volume, resulting in volume fractions of $3 \%$ and $5 \%$, respectively, for the scans shown in Figure 7B,D.

\section{4 | Manual segmentation of lymphatic vessels}

The equivalent OCT signal characteristics of the lymphatic and blood vessels in the ex vivo conjunctival tissue mean that they cannot be readily differentiated by the contrast in the $2 \mathrm{D}$ and $3 \mathrm{D}$ imaging methods presented here. In the context of examining tissue lymphatics, this ambiguity of the vessel types is problematic, but can be mitigated, to some extent, by additional image processing. Such processing can distinguish the lymphatics from blood vessels by the morphological features of the lymphatics, such as uneven vessel diameter and the frequent presence of valve structures. Based on this approach, we manually segmented a subset of the lymphatic vessel network in the scan shown in Figure 8A from Sample 7. This method first selects a large lymphatic vessel with obvious uneven vessel diameters in the $2 \mathrm{D}$ vessel projection image and with valve structures in the original 3D OCT scan. It then initiates vessel tracing from the selected lymphatic vessel by identifying the connecting vessels backward toward the smaller lymphatic vessels. The vessel connections are first identified from the $2 \mathrm{D}$ projection vessel image in Figure 8A, and then interpreted in the original 3D scan using the presence and orientation of the valve leaflets. If the connecting vessels do not have the matching depth or do not present valves in the connecting voxels, the connection is ruled out. The tracing is iterated and completed until all distal vessels present no more positive connections.

The identified network of connecting lymphatic vessels is then segmented by masking all other vessels in the image of the combined vessels. Figure 8B shows the resulting projection image of the segmented subset of the lymphatic vessel network. The flow direction of the lymph in the original in vivo tissue is also indicated by the red and yellow arrows toward the large lymphatic vessels. In addition, the red dots in Figure 8B mark the distal endpoints of the vessels, as visualized by OCT imaging. These examples may represent the initial lymphatics, but further study will be needed to confirm this or otherwise.

\section{5 | OCT imaging of aqueous vein plexus in sclera}

The aqueous humor is a transparent liquid and, in normal human eyes, drains via the trabecular meshwork into Schlemm's canal. The canal then directs the aqueous humor to a network of aqueous veins in the conjunctiva and aqueous venous plexuses and, finally, to the veins. The balance of this outflow and the production of aqueous humor regulates the IOP. The transparency of the aqueous humor led to the observation of the aqueous vein plexus in several vessel 

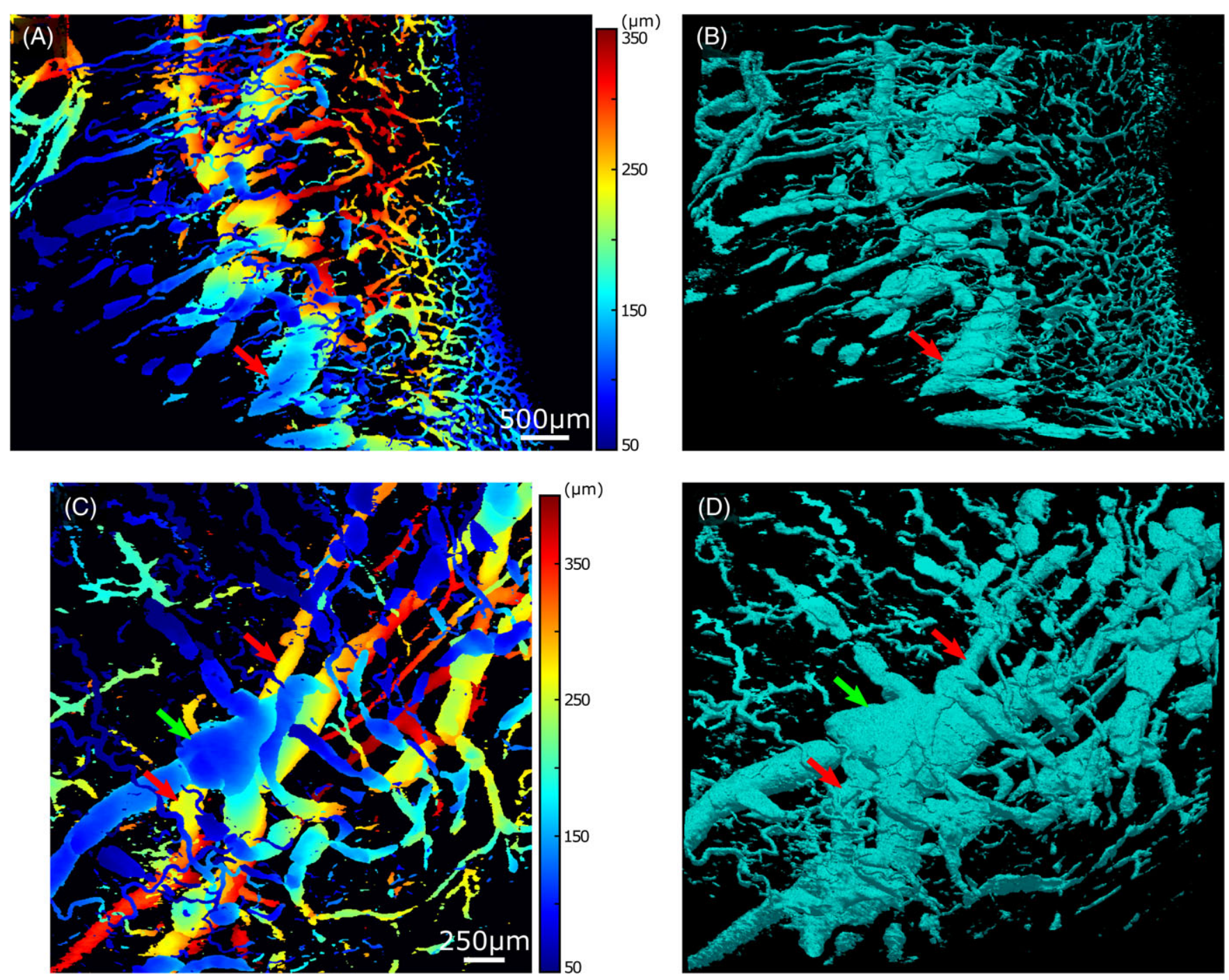

FIGURE 7 OCT imaging of conjunctival vessels in 3D. (A) Color-coded depth of the top surface of the vessels in Figure 1(B) of Sample 1. (B) Rendering of the 3D vessels in (A) from Visualization 1 (Video S1). Red arrows mark the same flat vessel. (C) Color-coded depth of the top surface of the vessels in Figure 4 of Sample 4. (D) Rendering of the 3D vessels in (C) from Visualization 2 (Video S2). Red arrows mark two connecting vessels below the vessel marked by the green arrows

images in this study. To separate and image these vessels with high fidelity, we excised the conjunctival tissue of one porcine eye (Sample 8). The conjunctiva has a thickness of $\sim 240 \pm 50 \mu \mathrm{m}$ [28]. We performed our OCT imaging on the remaining tissue. Figure 9 presents the results after removal of the conjunctival layer in the middle region (where the OCT FOV is indicated by the blue rectangle in Figure 9A). The aqueous vein plexus at this location shows notable pigmentation of the surrounding tissue (seen in the photo, Figure 9A) with vessels matching those imaged in Figure 9B. In contrast to the lymphatic vessels, the aqueous vein plexus does not present valve structures, and presents even diameters along the individual vessel segments. Compared to superficial blood vessels in the conjunctiva, these vessels overall have larger diameters and more branch points with prevalent local open loops, as indicated by the dashed line in Figure 9B. These differences, together with the typically larger depths of the aqueous vein plexus underlying the conjunctiva, will allow their ready isolation in future in vivo OCT lymphangiography imaging.

\section{4 | DISCUSSION}

This study has presented proof-of-principle imaging of conjunctival vessels ex vivo to elucidate vessel morphology, indicating the varying distribution and density of patent lymphatics in a range of porcine eyes and over a range of locations. It adds conjunctival lymphatics to the currently limited suite of results available from the emerging OCT lymphangiography techniques for label-free volumetric imaging studies. The first use of OCT lymphangiography for imaging the conjunctiva was presented by $\mathrm{Li}$ et al. on a patient with ocular graft-vs-host disease as an in vivo case study [19]. They observed several lymphatic vessel segments exhibiting dilation compared to those in normal subjects. Recently, Horstmann et al. showed the presence of lymphatic vessels 

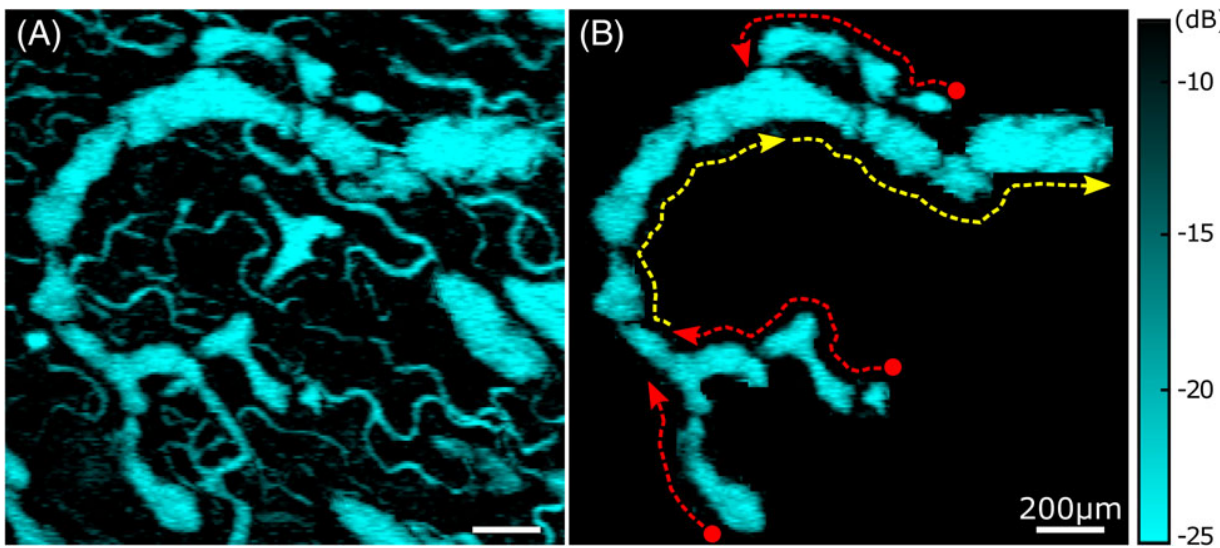

FIGURE 8 Manual segmentation of conjunctival lymphatic vessels in Sample 7. (A) Projection image of the blood and lymphatic vessels from the surface to $250 \mu \mathrm{m}$ deep. (B) A manually segmented subset of the lymphatic vessel network. Arrows: Expected flow trajectory in the lymphatic vessel. Scale bars: $200 \mu \mathrm{m}$

in vivo as local regions of very low signal in the highresolution OCT images of the mouse cornea, which is typically very close to the conjunctiva, with further image processing to segment several individual lymphatic vessel segments limited to a small FOV $(0.5 \times 0.5 \mathrm{~mm})$ [29]. In contrast, we have demonstrated conjunctival vessel imaging ex vivo, as a first step that avoids the complexity of in vivo dynamic tissue imaging, and aided by our recently proposed OCT lymphangiography imaging method [17]. We exploited the optical transparency (ie, lack of scattering) of the ex vivo blood and lymphatic vessels and segmented both vessel networks in 2D projections and in 3D volumetric images. Such imaging helps build a reference data set of vessel morphology for future studies of conjunctival lymphatics in general.

More particularly, this work represents a step in our development of OCT lymphangiography for in vivo imaging of glaucoma patients, particularly for guiding and assessing filtration surgery. Filtration surgery regulates the elevated IOP by creating an additional drainage pathway for the aqueous humor through a small channel, directing it to the subconjunctival tissue. For example, the commercially available $\mathrm{XEN}^{\circledR}$ technique builds this channel by implanting a gel stent with lumen diameter of $45 \mu \mathrm{m}$. In such surgeries, the integrity and density of conjunctival lymphatics have been suggested as key for the longevity of the created drainage pathway and in determining the treatment outcome [8], which has modest rates of successful IOP control of $48 \%$ and $40 \%$, respectively, at 3 and 5 years post a single surgery $[30,31]$. To improve the treatment outcome, one potential application of OCT lymphangiography is to image the conjunctival lymphatics pre-treatment to guide the selection of regions for surgery to those with healthy lymphatics that facilitate drainage. As a follow-up, OCT lymphangiography could be applied to image the conjunctival lymphatics posttreatment to investigate their role in the created drainage pathway and their association with the treatment outcome. The non-invasive, high-resolution, label-free imaging capability of OCT lymphangiography makes it a promising candidate for such applications. Our follow-up work will include the translation of the OCT lymphangiography technique from ex vivo to in vivo imaging toward its adoption for guiding and assessing glaucoma filtration surgery. Additionally, such a label-free volumetric imaging technique holds potential for applications to various other fields, such as neuroimmunology [32].

The vessel images from ex vivo porcine conjunctival and underlying tissue showed the presence of lymphatic vessels, regular blood vessels and the aqueous vein plexus. We
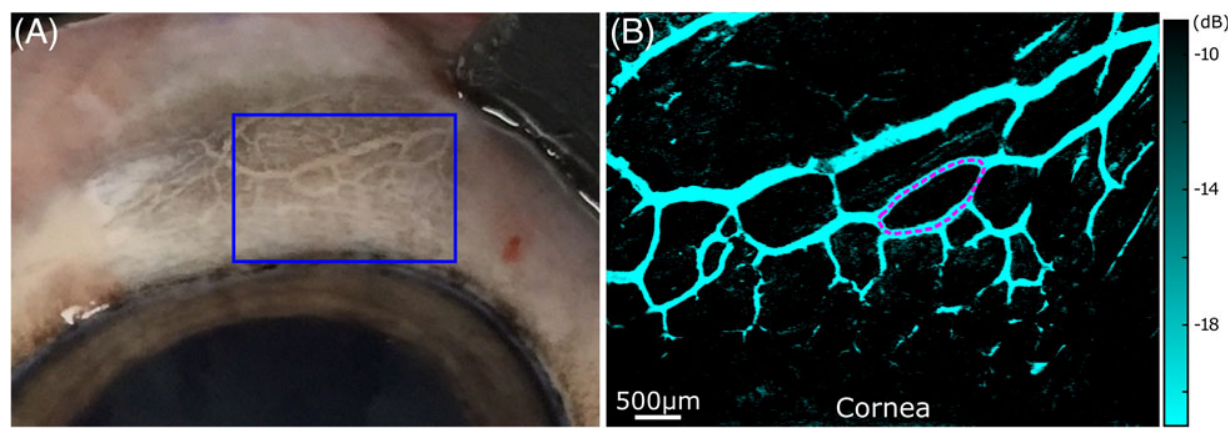

FIGURE 9 OCT imaging of vessels underlying the conjunctiva in Sample 8. (A) Photograph of the tissue after removal of the conjunctival tissue with the vessels in a background of noticeable pigmentation. Blue rectangle shows region in OCT image in (B). (B) Projection image of vessels from the surface to $150 \mu \mathrm{m}$ deep, with the position of the cornea marked by the label. The dashed line shows a local open loop 
observed their different morphological features, with the lymphatics characterized by thick uneven vessels and prevalent valve structures, regular blood vessels presenting as thin even vessels, and deep aqueous vein plexus presenting as large vessels with more branch points and local open loops. These features allow qualitative differentiation of vessels, especially for the mixed blood and lymphatic vessels, as shown in the proof-of-principle example in Section 3.4. However, differentiating vessels directly from the OCT signal alone is not possible, as they all show transparency and low signal levels ex vivo. This limitation should be largely overcome when OCT lymphangiography is transferred to in vivo conjunctival imaging based on our previous approach [17]. The regular blood vessels in the in vivo conjunctival tissue will have higher OCT signal than ex vivo, and can be separated using speckle decorrelation weighted by the corresponding OCT signal [17]. The aqueous veins can still have indistinguishable OCT signal from the lymphatics in vivo, but can be separated from lymphatics by their appearance mainly at greater depths. The separated vessel types can be color coded to allow clear differentiation and visualization of different vessel networks.

A limitation of the current study is the lack of validation of the segmented vessel structures. We have attempted to obtain histology using $5^{\prime}$-nucleotidase (5'-Nase) for staining the conjunctival lymphatic vessels and alkaline phosphatase (ALPase) for blood vessels to validate OCT images. A limitation is that most of Tenon's capsule has to be removed resulting in only lymphatic capillaries being presented in histology, because they are located superficial to Tenon's capsule. The lymphatic pre-collectors are mostly located deeper than Tenon's capsule, and are, thus, not present in the histological specimen. However, our current OCT lymphangiography described in this study can reliably image precollectors, but not initial lymphatics yet. Therefore, we were unsuccessful in fully validating our imaging method by direct comparison with histology [8].

OCT lymphangiography, as an emerging family of imaging techniques, mainly uses the markedly low OCT signal in the lymphatic vessels for segmentation. Thus, the imaging resolution of the system is critical in enabling the capture of the initial lymphatics (capillaries), which are important for draining the interstitial fluid and can be as small as $10 \mu \mathrm{m}$ in diameter. The OCM used in this study provides highimaging resolution (axial: $1.5 \mu \mathrm{m}$ in air; transverse: $1.6 \mu \mathrm{m}$ ) to resolve the small vessel structures, and the residual blood cells in the vessels. However, for in vivo clinical applications, it is limited by its small FOV, impeding the examination of the large vessel network, and its very short working distance makes imaging challenging. With the resolution of the OCT scanner used in this study (axial: $5.5 \mu \mathrm{m}$ in air; transverse: $13 \mu \mathrm{m}$ ), our OCT lymphangiography method clearly resolved vessels with diameters as small as 15 to $20 \mu \mathrm{m}$. The observation of valve structures in the segmented lymphatic vessels indicates that the collecting lymphatic vessels in the conjunctiva can be clearly visualized. However, the capability of our current OCT system to resolve the blind ends of the small lymphatic capillaries is still questionable. Though we did observe instances of the blind ends of vessels, as in Figure 8, it is not clear that we can do this reliably. The lumens of these ex vivo capillaries might be smaller than their in vivo equivalents or may even have collapsed in the ex vivo tissue. When the lumen size is smaller than the imaging resolution, the vessel voxels are impacted by partial-volume effects, combining portions of low signal in vessel voxels with the high signal from the neighboring tissue.

\section{5 | CONCLUSION}

In this paper, we have presented a preliminary study on OCT lymphangiography imaging of healthy porcine conjunctiva ex vivo, based on OCT and high-resolution OCM. The thick uneven lymphatic vessels exhibited optical transparency to the near-infrared 785-nm and 1300-nm light, resulting in low-backscattering signals that approached the noise level of the OCT and OCM systems used. The thin even blood vessels exhibited a lack of blood and blood flow inside the vessel lumen and, thus, produced a low OCT signal closely equivalent to that of the lymphatic vessels. To segment these vessels, our OCT lymphangiography method first estimated and compensated for the attenuation in the OCT signal using the single-scattering model and then separated the vessels from surrounding tissue into $2 \mathrm{D}$ projection and $3 \mathrm{D}$ volumetric images. The images allow the visualization of the vessel morphologies with the prevalent valve structures identifying the lymphatic collecting vessels. In addition, the blood vessels and aqueous vein plexus in the OCT lymphangiography images potentially confound the identification of the lymphatic vessels. However, we demonstrated that the separation of the lymphatics from the other vessels is feasible by additional image processing using the characteristic vessel shapes. The visualization of these conjunctival vessels in our preliminary study demonstrated the feasibility of OCT lymphangiography technique for ex vivo imaging, and provides a valuable reference on vessel morphology for subsequent in vivo label-free imaging of lymphatic vessels toward guiding and monitoring glaucoma filtration surgery.

\section{ACKNOWLEDGMENTS}

The authors thank Macdara O. Murchu for assistance in clinical scanning at the Lions Eye Institute. P.G. was supported by a Healy Research Collaboration Award from the Healy Medical Research Foundation. This study was supported with funding from the Australian Research Council, the National Health and Medical Research Council, and the 
Government of Western Australia, Department of Health, Research Translation Projects program.

\section{ORCID}

Peijun Gong (D) http://orcid.org/0000-0003-1888-2438

Karol Karnowski (D) http://orcid.org/0000-0002-5426-5878

David D. Sampson (D) http://orcid.org/0000-0001-6724-3873

\section{REFERENCES}

[1] M. A. Swartz, Adv. Drug Deliv. Rev. 2001, 50, 3.

[2] D. Negrini, A. Moriondo, J. Physiol. 2011, 589, 2927.

[3] F. Zhang, G. Niu, G. Lu, X. Chen, Mol. Imaging Biol. 2011, 13, 599.

[4] T. F. O'Donnell, J. C. Rasmussen, E. M. Sevick-Muraca, J. Vasc. Surg. Venous Lymphat. Disord. 2017, 5, 261.

[5] B. Zhu, E. M. Sevick-Muraca, Br. J. Radiol. 2015, 88, 20140547.

[6] L. L. Munn, T. P. Padera, Microvasc. Res. 2014, 96, 55.

[7] E. I. Galanzha, M. S. Kokoska, E. V. Shashkov, J. W. Kim, V. V. Tuchin, V. P. Zharov, J. Biophotonics 2009, 2, 528.

[8] D. Y. Yu, W. H. Morgan, X. Sun, E. N. Su, S. J. Cringle, P. K. Yu, P. House, W. Guo, X. Yu, Prog. Retin. Eye Res. 2009, 28, 303.

[9] H. A. Quigley, A. T. Broman, Br. J. Ophthalmol. 2006, 90, 262.

[10] C. L. Chen, R. K. Wang, Biomed. Opt. Express 2017, 8, 1056.

[11] R. A. Leitgeb, R. M. Werkmeister, C. Blatter, L. Schmetterer, Prog. Retin. Eye Res. 2014, 41, 26.

[12] B. J. Vakoc, R. M. Lanning, J. A. Tyrrell, T. P. Padera, L. A. Bartlett, T. Stylianopoulos, L. L. Munn, G. J. Tearney, D. Fukumura, R. K. Jain, B. E. Bouma, Nat. Med. 2009, 15, 1219.

[13] R. K. Wang, S. Hurst, Opt. Express 2007, 15, 11402.

[14] P. Gong, S. Es'haghian, F. M. Wood, D. D. Sampson, R. A. McLaughlin, Exp. Dermatol. 2016, 25, 722.

[15] P. Gong, S. Es'haghian, K. A. Harms, A. Murray, S. Rea, B. F. Kennedy, F. M. Wood, D. D. Sampson, R. A. McLaughlin, J. Biophotonics 2016, 9, 626.

[16] D. M. Sampson, P. Gong, D. An, M. Menghini, A. Hansen, D. A. Mackey, D. D. Sampson, F. K. Chen, Invest. Ophthalmol. Vis. Sci. 2017, 58, 3065.

[17] P. Gong, S. Es'Haghian, K. A. Harms, A. Murray, S. Rea, F. M. Wood, D. D. Sampson, R. A. McLaughlin, Biomed. Opt. Express 2016, 7, 4886.

[18] S. Yousefi, J. Qin, Z. Zhi, R. K. Wang, J. Biomed. Opt. 2013, 18, 086004.

[19] P. Li, Y. Sun, S. Hariri, Z. Zhou, Y. Inamoto, S. J. Lee, T. T. Shen, R. K. Wang, Quant. Imaging Med. Surg. 2015, 5, 163.

[20] Y. M. Liew, R. A. McLaughlin, P. Gong, F. M. Wood, D. D. Sampson, J. Biomed. Opt. 2013, 18, 061213.

[21] A. Zhang, Q. Zhang, R. K. Wang, Biomed. Opt. Express 2015, 6, 4130.
[22] M. Zhang, T. S. Hwang, J. P. Campbell, S. T. Bailey, D. J. Wilson, D. Huang, Y. Jia, Biomed. Opt. Express 2016, 7, 816.

[23] J. M. Schmitt, A. Knüttel, R. F. Bonner, Appl. Opt. 1993, 32, 6032.

[24] L. Scolaro, R. A. McLaughlin, B. R. Klyen, B. A. Wood, P. D. Robbins, C. M. Saunders, S. L. Jacques, D. D. Sampson, Opt. Express 2012, 3, 366.

[25] P. Gong, R. A. McLaughlin, Y. M. Liew, P. R. T. Munro, F. M. Wood, D. D. Sampson, J. Biomed. Opt. 2014, 19, 021111.

[26] A. Curatolo, M. Villiger, D. Lorenser, P. Wijesinghe, A. Fritz, B. F. Kennedy, D. D. Sampson, Opt. Lett. 2016, 41, 21.

[27] J. M. Lauweryns, L. Boussauw, Z. Zellforsch. Mikrosk. Anat. 1973, 143, 149.

[28] X. Zhang, Q. Li, B. Liu, H. Zhou, H. Wang, Z. Zhang, M. Xiang, Z. Han, H. Zou, Invest. Ophthalmol. Vis. Sci. 2011, 52, 7787.

[29] J. Horstmann, H. Schulz-Hildebrandt, F. Bock, S. Siebelmann, E. Lankenau, G. Hüttmann, P. Steven, C. Cursiefen, Invest. Ophthalmol. Vis. Sci. 2017, 58, 5880.

[30] R. R. Allingham, K. F. Damji, S. Freedman, S. E. Moroi, G. Shafranov, M. B. Shields, Shields' Textbook of Glaucoma, Lippincott Williams \& Wilkins, Philadelphia, PA 2005.

[31] K. Nouri-Mahdavi, L. Brigatti, M. Weitzman, J. Caprioli, Ophthalmology 1995, $102,1760$.

[32] A. Louveau, I. Smirnov, T. J. Keyes, J. D. Eccles, S. J. Rouhani, J. D. Peske, N. C. Derecki, D. Castle, J. W. Mandell, K. S. Lee, T. H. Harris, J. Kipnis, Nature 2015, 523, 337.

\section{SUPPORTING INFORMATION}

Additional supporting information may be found online in the Supporting Information section at the end of the article.

Video S1 Volumetric imaging of conjunctival vessels in Sample 1.

Video S2 Volumetric imaging of conjunctival vessels in Sample 4.

How to cite this article: Gong P, Yu D-Y, Wang Q, et al. Label-free volumetric imaging of conjunctival collecting lymphatics ex vivo by optical coherence tomography lymphangiography. J. Biophotonics. 2018;11:e201800070. https://doi.org/10.1002/jbio. 201800070 